\title{
Study on nonlinear conduction of PEDOT:PSS conducting polymer
}

\author{
R.S.Abd-Elghany, H.Khoder, R.Khalil and M.K.El-Mansy \\ Physics Dept., Faculty of Science, Benha Univ., Benha, Egypt \\ E.Mail: Rasha.abdelghani@fsc.bu.edu.eg
}

\begin{abstract}
In the present study, solid film of poly (3,4-ethylenedioxythiophene):polystyrenesulfonate, (PEDOT:PSS) has been prepared by casting its aqueous solution at room temperature and are characterized by using X-ray diffraction and differential scanning calorimeter. The temperature-dependent behavior of the ac and dc conductivity of the PEDOT:PSS film has been investigated. The temperature dependence and scan rate dependence of $\mathrm{J}-\mathrm{V}$ characteristic curves have been tested. The study of frequency dependence of ac conductivity in a wide range of temperature up to $443 \mathrm{~K}$, indicates that the dominant conduction mechanism in PEDOT:PSS film is Overlapping-Large Polaron Tunneling (OLPT). The study of the electrical conduction with applied voltage shows a reversible negative differential resistance (NDR) through the turnover point (T.O.P) for $\mathrm{Cu} / \mathrm{PEDOT}: \mathrm{PSS} / \mathrm{Cu}$ device. Threshold current density, $\mathrm{J}_{\mathrm{th}}$, and threshold voltage, $\mathrm{V}_{\text {th }}$ at T.O.P as well as NDR are strongly affected by ambient temperature up to $383 \mathrm{~K}$ and scan rate up to $3 \mathrm{~V} / \mathrm{sec}$. The turnover behavior has been explained according to a competition between high conduction due to schottky emission and NDR due to field-induced of electrical polarization in conduction paths.
\end{abstract}

Keywords: PEDOT:PSS, Impedance spectroscopy, Negative differential resistance, Switching memory, Scan rate.

\section{Introduction}

Today, all electronic devices in our life such as computers, health sensors, space technology and etc., are an integral component of electronic memory. This is the reason both researchers and the business community are attracting great interest in these products. In addition, the latest trend in electronics is moving from rigid to flexible electronic parts and appliances that are bendable. Organic semiconductor materials are the best candidates in this context of redefining memory technology to follow the new path in-memory technologies and applications. It should be pointed out that organic materials offer a wide range of advantages, such as being easy to process at low temperatures, a low weight, high mechanical flexibility, and it is simple to tunable the electrical and optical properties. Organic materials are being explored for a wide range of electronic devices such as light-emitted diode, photovoltaic cells, batteries, transistors, and memories, due to all the above-mentioned benefits. Recently, organic memory devices have attracted many interests as new devices to overcome restrictions of the scientific or fabrication process at inorganic semiconductor memory devices. The memory devices based on PEDOT:PSS polymer, which it is attracted much interest as it is easy to film fabrication, high conductivity, good stability, and electrical switching characteristics[1,2]. In general switching memory's characteristics are determined by two mechanisms [3-5]. The electrodelimited conduction (ELC) process, which involves Schottky emission and Fowler-Nordheim tunneling, is the first. The bulk-limited conduction (BLC) mechanism, which includes space charge limited conduction (SCLC) and Pool-Frenkel (P-F) emission, is the second form. The ELC mechanism is based on the electrical nature of electrodematerial interfaces, while the BLC mechanism is based on the material's electrical properties. The conduction mecha- nism of switching memory based on PEDOT:PSS film between two metal electrode was generally explained by charge trapping and cation movement [6,7], the formation and rupture of conductive filaments $[8,9],[10-12]$, to hole injection and resonant tunneling, hole blockage and extraction, and ionic motion in the PEDT:PSS film [13] and change of the electrical conductivity of PEDOT:PSS due to electrical field induced dipolar reorientation. [14].

In this paper, we report a unipolar switching and reversible behavior in PEDOT:PSS active film between two copper electrodes as $\mathrm{Cu} / \mathrm{PEDOT}: \mathrm{PSS} / \mathrm{Cu}$ structure device. We study the change in the electrical conductivity of PEDOT:PSS film under different temperatures. The (J-V) characteristic curves are investigated under different temperatures and different scan rates. The negative differential resistance is explained due to electrical field-induced of dipolar reorientation and trapping.

\section{Experimental details}

An aqueous solution of poly(3,4 ethylene dioxythiophene) doped with poly(styrene sulfonic acid) (PEDOT:PSS 1.2wt\%, Baytron/ CleviosPH500) is purchased from Heraeus, The dark blue solution was filtered through a $1 \mu \mathrm{m}$ filter to produce a more homogenous solution; after that, it was direct cast in a Petri - plastic dishes and left for one week at room temperature to dry obtaining solid film of PEDOT:PSS. The structure of the prepared PEDOT:PSS film was characterized by a diffractometer type XRD (BRUKER model D8 advance). X-ray diffractometer using monochromatized $\mathrm{CuK} \alpha$ radiation of wavelength $(\lambda) 1.540$ $\AA$ from a fixed source operated at voltage $40 \mathrm{kV}$ and current $40 \mathrm{~mA}$. XRD was investigated between $2 \theta$ angles range $4-70$ degrees. Bragg's law $(\mathrm{n} \lambda=2 \mathrm{~d} \sin \theta)$, where $\mathrm{n}$ is an integer, was used to calculate the crystallographic spacing (d). The thermal behavior of the sample was examined 
using a SETARAM TG-DSC16 Digital Scanning Calorimeter (DSC). The sample was heated from 300 to $700 \mathrm{~K}$ at a heating rate $20 \mathrm{~K} / \mathrm{min}$. The PEDOT:PSS film of thickness about $30 \mu \mathrm{m}$ is used to study ac electrical conductivity by using $8110 \mathrm{G}$ programmable automatic RCL GWINSTEK meter in the frequency range $20 \mathrm{~Hz}$ to $10 \mathrm{MHz}$ at different temperatures. Two copper electrodes have been used to measure J-V characteristics by using Keithley Source Meter type $2420 \mathrm{C}$, at different temperatures and different scan rates. The dc conductivity of sandwiched film between two copper electrodes is measured by using Keithley source at constant applied voltage, and recording the current of the sample with temperature. The dc conductivity was calculated using the following equation, $\sigma=\frac{\mathrm{I}}{\mathrm{V}} \frac{\mathrm{t}}{\mathrm{A}}$, where $\mathrm{I}$ is the electric current, $\mathrm{V}$ is the applied voltage, $\mathrm{t}$ is the thickness of the sample and $\mathrm{A}$ is the cross sectional area.

\section{Results and discussion}

$\mathrm{X}$-ray diffraction (XRD) investigation is used to infer and characterize the micro structural of PEDOT:PSS polymer. It is also extended to clarify the degree of the crystallinity of the polymer. Diffraction patterns for PEDOT:PSS film under investigation are shown in Fig (1). The five peaks at $2 \theta$ values of approximately at $6.2^{\circ}, 18.7^{\circ}$, $25.3^{\circ}, 26.4^{\circ}$, and $28.37^{\circ}$ in PEDOT:PSS film correspond to the lattice d spacing calculated in terms of Bragg's law is shown in Fig (1). The diffraction at $6.2^{\circ}$ was attributed to the lamella stacking between two distinct alternate ordering PEDOT:PSS and $18.7^{\circ}$ was attributed to inter-chain ring stacking between PEDOT:PSS[15,16]. This lamella stacking distance agrees well with the widths of the PEDOT and PSS chain, which are $14.2 \mathrm{~A}^{\circ}$, estimated according to chemical structural simulation[17].On the other hand, the peaks at $25.3^{\circ}, 26.4^{\circ}$, and $28.37^{\circ}$ can be attributed to the distances between $\pi-\pi$ stacking (010) of the PEDOT chains $[18,19]$. The result confirmed that XRD pattern is similar to pristine PEDOT:PSS.

The differential scanning calorimetric (DSC) is an important technique that was used to determine thermal transition temperatures. PEDOT:PSS is one of the partially crystalline polymers exhibiting two endothermic peaks as shown in Fig (2).The first peak that appeared at about 383 $\mathrm{K}$ can be attributed to the dehydration $\left(\mathrm{T}_{\mathrm{d}}\right)$ of adsorbed water in the polymer[19]. The second peak that appeared at about $603 \mathrm{~K}$ can be assigned to melting temperature, $\left(\mathrm{T}_{\mathrm{m}}\right)$. PEDOT:PSS does not have a glass transition value due to the strong ionic bonding interaction between $\mathrm{PEDOT}^{+}$and $\operatorname{PSS}^{-}[19]$.

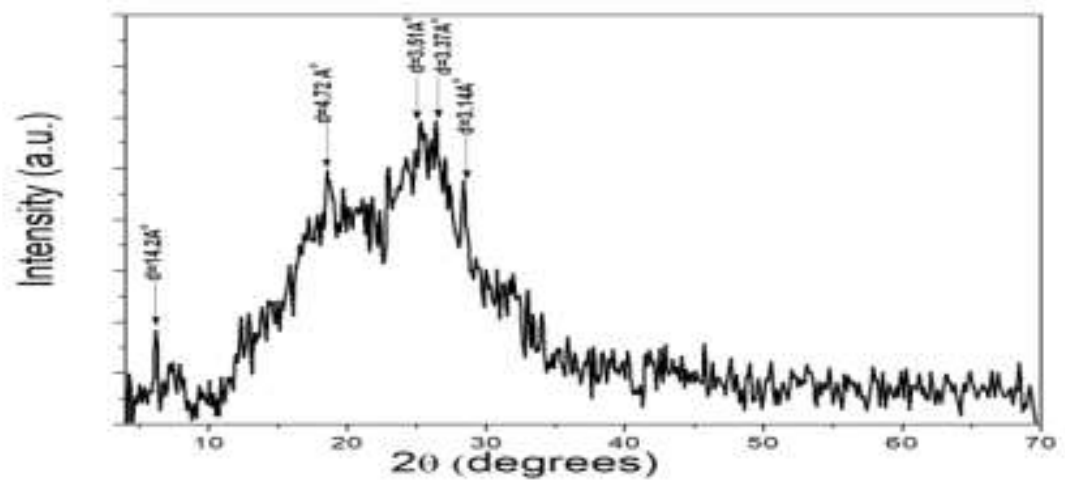

Fig (1) XRD pattern for PEDOT: PSS film.

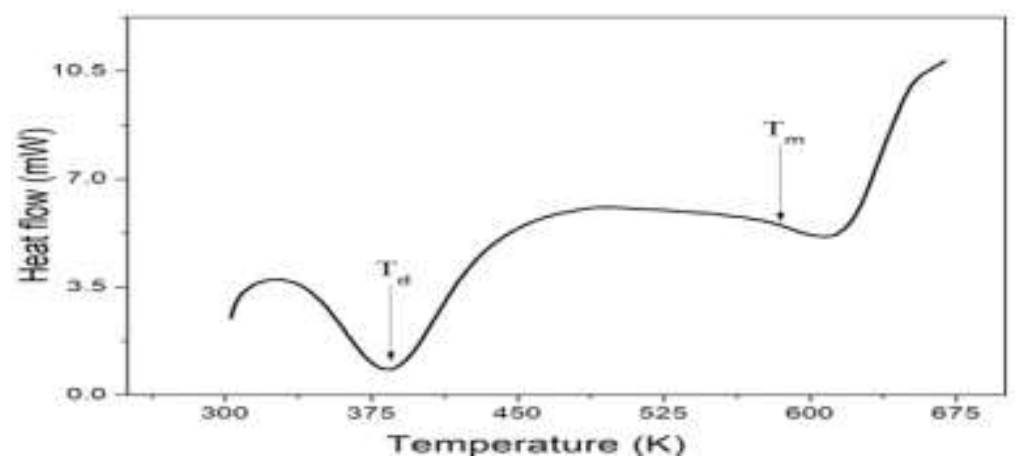

Fig (2) Schematic DSC curve of amount of heat flow versus temperature for PEDOT: PSS film. 
Mansy

The frequency dependence of the total conductivity $\left(\sigma_{\text {tot }}\right)$ for the PEDOT:PSS film was studied at different temperatures, show Fig (3), which follows the universal power law[20]:

$$
\sigma_{\text {tot }}(\omega)=\sigma_{\mathrm{s}}+\mathrm{A} \omega^{\mathrm{S}}
$$

Where, $\sigma_{\mathrm{s}}$ is the frequency-independent parameter which refers to the stationary conductivity (the extrapolation of the plateau region to zero frequency), $\omega$ is the angular frequency, $\mathrm{s}$ is the frequency power exponent and $\mathrm{A}$ is frequency independent pre-power factor.

Fig (3), illustrates that, the ac conductivity increases with increasing the frequency and almost independent at low frequency. So the ac conductivity curves can be divided into two parts. In the first part, the conductivity has a nearly constant value at the lower frequency region which corresponds to stationary conductivity. The stationary conductivity increases with increasing of temperature indicat- ing that the conduction process is thermally activated. The second part, the dispersion region, the conductivity increases with increasing the frequency at a high-frequency region, which obeys the universal power law, $\sigma_{\mathrm{ac}} \alpha \omega^{\mathrm{s}}$. The strong frequency dependence of ac conductivity can be described by the second term in Eq (1). The values of the frequency exponent ( $\mathrm{s}$ ) have been attained from the linearfit data of ac conductivity spectra in high frequency dispersion region, which lies in the range $0.34<\mathrm{s}<1$, as shown in inset Fig (3). Additionally, it can be noticed that, the exponent $\mathrm{s}$ decreases with increasing temperature and then increase at a minimum value at $363 \mathrm{~K}$. Accordingly, these results lead to the prediction that the domination conduction mechanism is the Overlapping Large Polaron Tunneling (OLPT), which is the most suitable model to characterize the electrical conduction mechanism in PEDOT:PSS film [21-24].

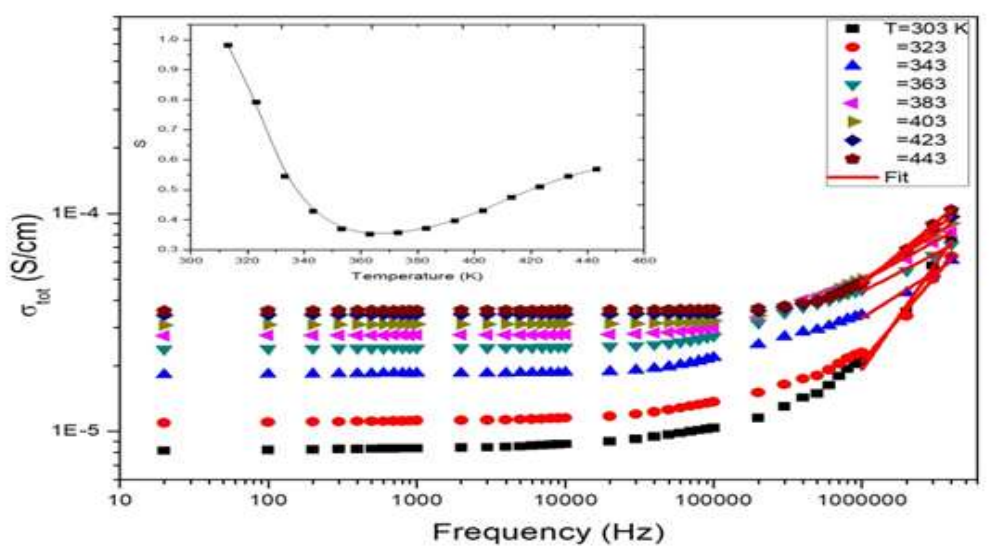

Fig (3) Variation of the total conductivity $\sigma_{\text {tot }}$ with frequency at different ambient temperatures for PEDOT:PSS film.

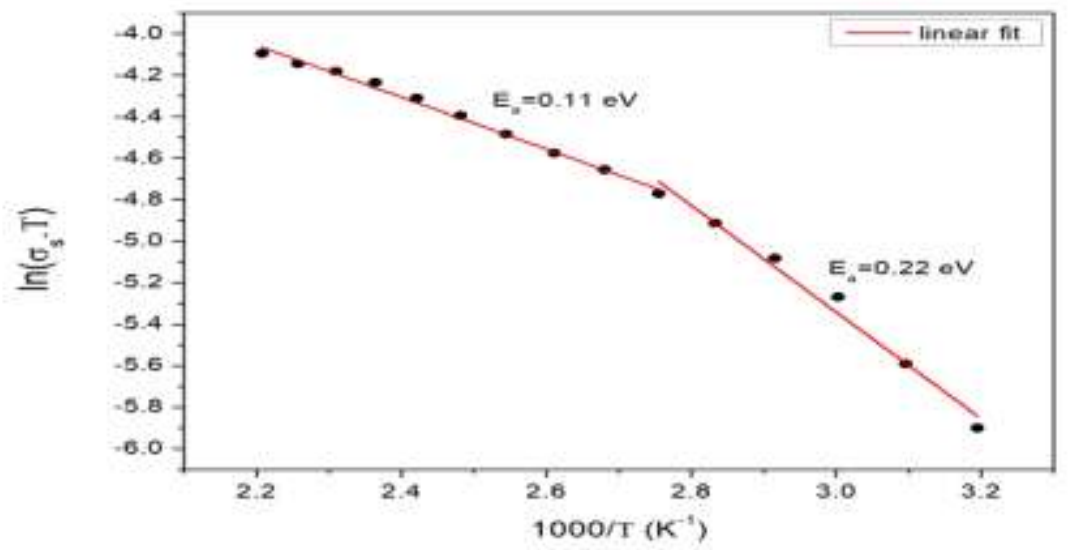

Fig (4) Temperature dependence of stationary conductivity at $\mathrm{f}=0$ for PEDOT:PSS film. 
Fig (4), shows the temperature dependence of $\ln \left(\sigma_{s} T\right)$ against $1000 / \mathrm{T}$ at $\mathrm{f}=0$ which can be described by the following Arrhenius equation[25].

$$
\sigma_{s} T=A \exp \left(\frac{-E_{a}}{k_{B} T}\right)
$$

Where $E_{a}$ is the activation energy for conduction in PEDOT:PSS polymer, A is temperature independent constant, which depends on the physical and chemical properties of the polymer matrix and $\mathrm{k}_{\mathrm{B}}$ is Boltzmann's constant. The behavior is divided into two regions with thermal activated.

The conductivity increases with increasing the temperature due to an increased polymer chain motion, which produces an increase in the free volume of the system [26].
As the amorphous degree in matrix increases the polymer chain acquires faster internal modes in which bond rotations produce polymer chain motion to support inter and intra-chain ion hopping and thus the conductivity increases. The values of activation energies have been extracted using the least square fitting of Eq (2). The value of activation energy in low temperature range is $0.22 \mathrm{eV}$ and decreases in high temperature range to $0.11 \mathrm{eV}$.

The dc conductivity as a function of temperature for PEDOT:PSS film between two copper electrodes (Cu/PEDOT:PSS/Cu device). The device was measured at low voltage, 1V. Fig (5), illustrates the temperature dependence of dc conductivity. The activation energy is calculated by Arrhenius equation (2), its value is $\mathrm{E}_{\mathrm{dc}}=0.12 \mathrm{eV}$.

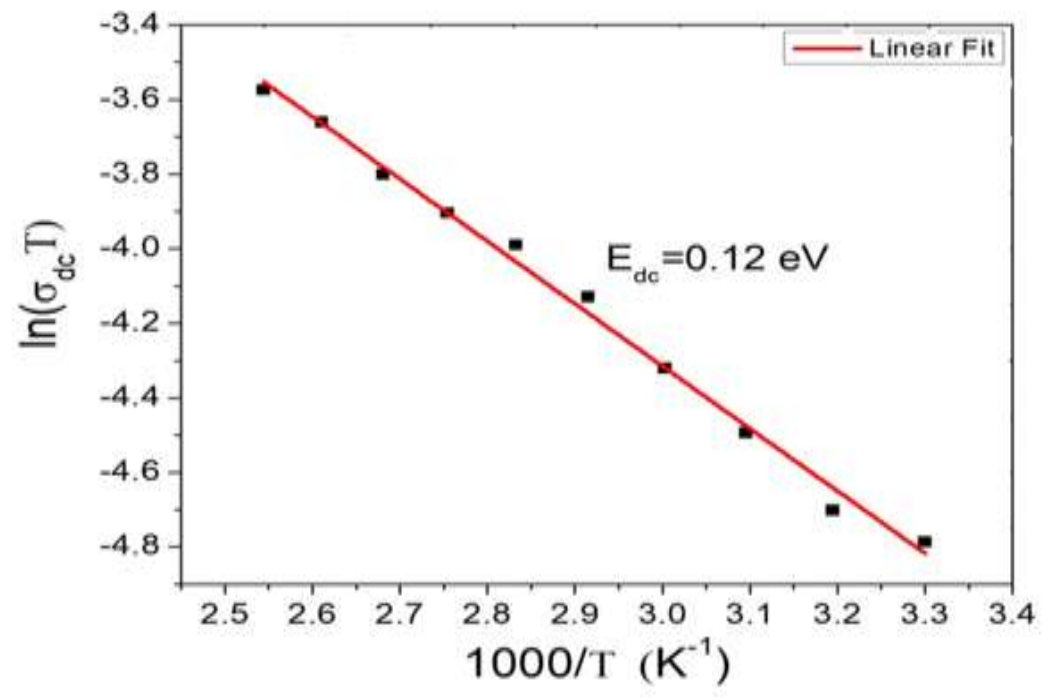

Fig (5) Temperature dependence of dc conductivity for $\mathrm{Cu} / \mathrm{PEDOT}$ :PSS/Cu device.

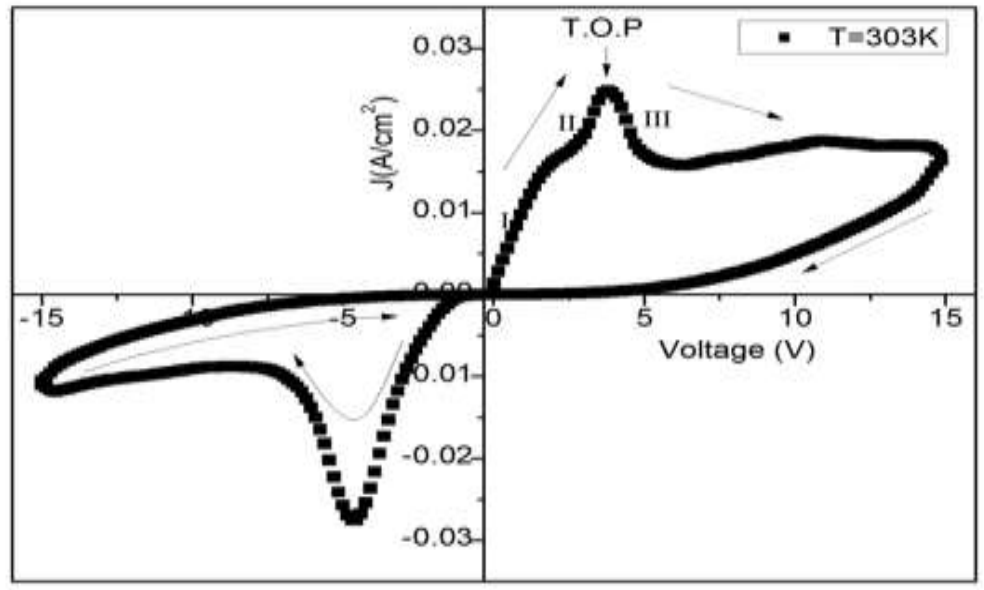

Fig (6) J-V characteristic curve of $\mathrm{Cu} / \mathrm{PEDOT}$ : PSS/Cu device at room temperature. 
Fig (6), illustrates J-V characteristic curves for $(\mathrm{Cu} / \mathrm{PEDOT}: \mathrm{PSS} / \mathrm{Cu}$ device$)$ at room temperature. The general behavior is divided into three parts; namely low resistance state (ON state), turnover point (T.O.P), and negative differential resistance (NDR. When the applied voltage scans from 0 to $+15 \mathrm{~V}$, the current density increases linearly (region I). By increasing the applied voltage, the current density increases exponentially (region II). The current density reaches its maximum value at a turnover point (T.O.P). By increasing the applied voltage beyond a turnover point, the current density drops gradually with appearing negative differential resistance (NDR). The resistance state is translated from a low-resistance state (ON state) to a high resistance state (OFF state). By decreasing the sweep voltage from $+15 \mathrm{~V}$ to 0 , the current density decreases gradually (OFF state) and forming a hysteresis area between go and back sweep in the forward direction (positive polarity). At the reverse direction (negative polarity), the current density reincreases with increasing applied voltage as similar behavior to that of the positive polarity. $\mathrm{J}-\mathrm{V}$ characteristic curves exhibit reversible a unipolar resistive switching memory with appearance of negative differential resistance.

The charge conduction mechanism can be qualitatively explained in positive voltage direction by using the concept of Schottky barrier with a combined effect of a hole injection (tunneling) and electrical polarization in the polymer. The interfaces between $\mathrm{Cu}$ and PEDOT:PSS form Schottky junctions with symmetric barrier heights $\left(\varphi_{\mathrm{B}}\right)$ and depletion region. In the low voltage, the current density of the injection (tunneling) of holes from electrode to HOMO level of PEDOT:PSS increases with an ohmic behavior at region I, Fig (7a). At the applied voltage increases the holes have enough energy provided by thermal activation, so the holes in the metal will overcome the energy barrier at the metal PEDOT:PSS polymer interface to inject to HOMO level of PEDOT:PSS and the current density increases rapid with an expentional behavior (regionII) before reaching to turn- over point (T.O.P) at threshold voltage $\left(\mathrm{V}_{\mathrm{th}}\right)$. The exponential dependence of region II in J-V curve can be explained by schottky emission through a barrier having height $\varphi_{\mathrm{B}}$. The schottky emission mechanism can be described by following equation[5].

$$
J=A^{*} T^{2} \exp \left[\frac{-\mathrm{q}\left(\varphi_{B-} \sqrt{\frac{V}{4 \pi \varepsilon d}}\right)}{\mathrm{K}_{\mathrm{B}} \mathrm{T}}\right]
$$

Where, $\mathrm{J}$ is the current density, and $\mathrm{V}$ is the applied potential, $T$ is measurement temperature, $A^{*}(120 \mathrm{~A}$ $\mathrm{cm}^{-2} \mathrm{~K}^{-2}$ ) is the Richardson constant, $\varphi_{\mathrm{B}}$ is barrier height, $\mathrm{k}_{\mathrm{B}}$ is Boltzmann constant, $\varepsilon\left(=\varepsilon_{0} \varepsilon_{\mathrm{r}}\right)$ is the dynamic dielectric constant and $d$ is the thickness of sample. The region (II) can be fitted by Eq (3), Fig (7b).

In negative differential resistance (NDR), region (III), the reduction of current density with increasing the applied voltage is attributed to a part of the injected charge carriers are trapped at $\mathrm{PEDOT}^{+}$sites of the lattice structure. In addition, the rotation of electric dipoles in the polymer under influence electric voltage at turnover point produces an inverse electric field, which leads to a decrease of the resultant field on the device. The competition of conduction due to carrier injection in the region (II) and that reduction of conductance at region III lead to a turnover behavior. The negative differential conductance (NDC) at region III can be explained by the following empirical Eq (4) as shown in Fig (7c).

$$
\mathrm{G}=\mathrm{G}_{\mathrm{o}} \exp \left(-\frac{\mathrm{F}}{\mathrm{F}_{o}}\right)^{0.5}
$$

Where $G_{o}$ is the ultimate value of conductance before T.O.P and $\mathrm{F}_{\mathrm{o}}$ is a characteristic field.

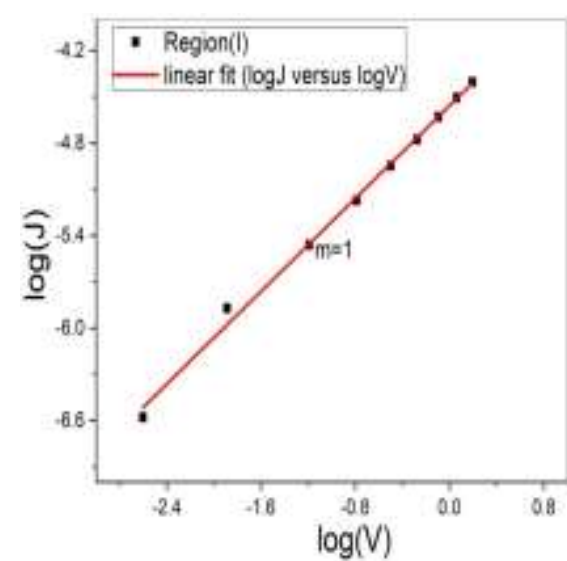

(a)

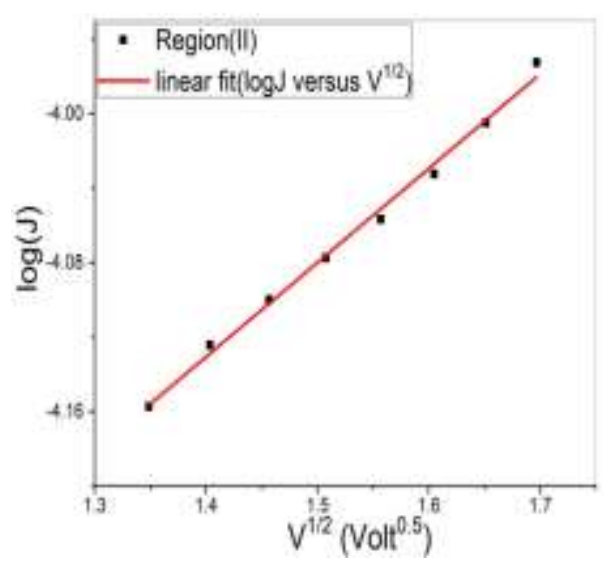

(b)

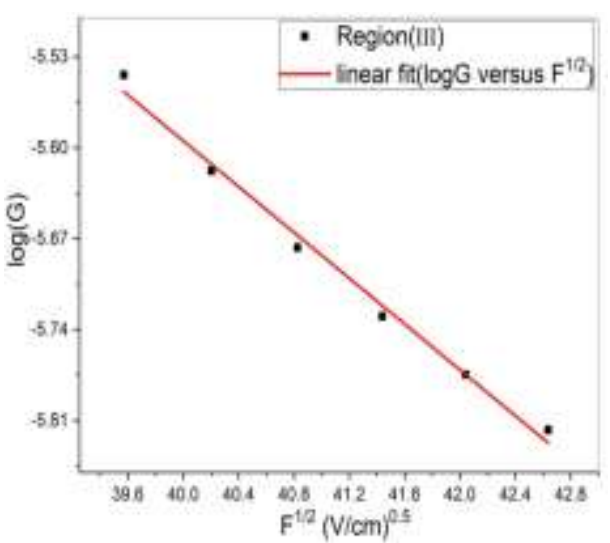

(c)

Fig (7) (a) The logarithmic scale of current density (J) versus V, (b) semi logarithmic scale of current density (J) versus $\mathrm{V}^{1 / 2}$ and (c) semi logarithmic scale of conductance $(\mathrm{G})$ versus $\mathrm{F}^{1 / 2}$ for Cu/PEDOT:PSS/Cu device 
Fig (8), illustrates J-V characteristic curves for $\mathrm{Cu} / \mathrm{PEDOT}: \mathrm{PSS} / \mathrm{Cu}$ device at different temperatures. The schottky emission mechanism is applied for region (II) at different temperatures and the barrier height and the dielectric constant are extracted and tabulated in table (1).

The values of both $\mathrm{J}_{\text {th }}$ and $\mathrm{V}_{\text {th }}$ obtained at turnover point (T.O.P) are recorded and plotted versus temperature in forward and reverse directions, Fig (9). It is clear the turnover point in the forward direction is shifted towards higher threshold current density and higher threshold voltage as the ambient temperature was increased, Fig (9a). Whereas in the reverse direction, the turnover point starts to inhibit after $\mathrm{T}=363 \mathrm{~K}, \mathrm{Fig}(9 \mathrm{~b})$. The increasing of both $\left(\mathrm{J}_{\text {th }}\right.$ and $\left.\mathrm{V}_{\mathrm{th}}\right)$ with increasing the temperature is attributed to increasing of injection or tunneling of holes PEDOT:PSS polymer in positive polarity, and extraction in the negative polarity.

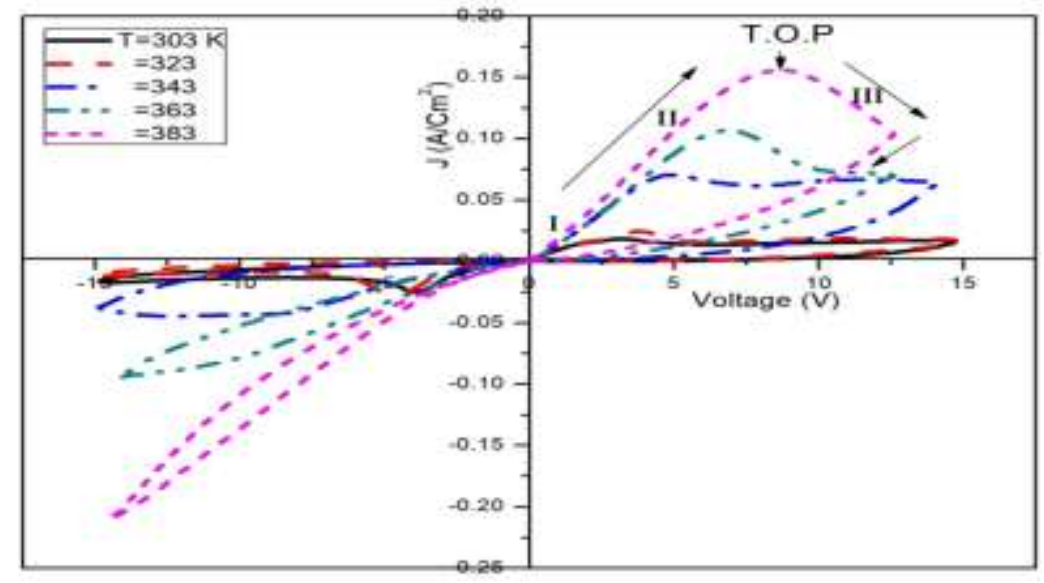

Fig (8) J-V characteristic curve of $\mathrm{Cu} / \mathrm{PEDOT}$ : PSS/Cu device at different temperatures.

Table (1) The deduced values of fitting parameters $\Phi_{\mathrm{B}}(\mathrm{eV})$ and $\varepsilon$ for $\mathrm{Cu} / \mathrm{PEDOT}: \mathrm{PSS} / \mathrm{Cu}$ device at different temperatures.

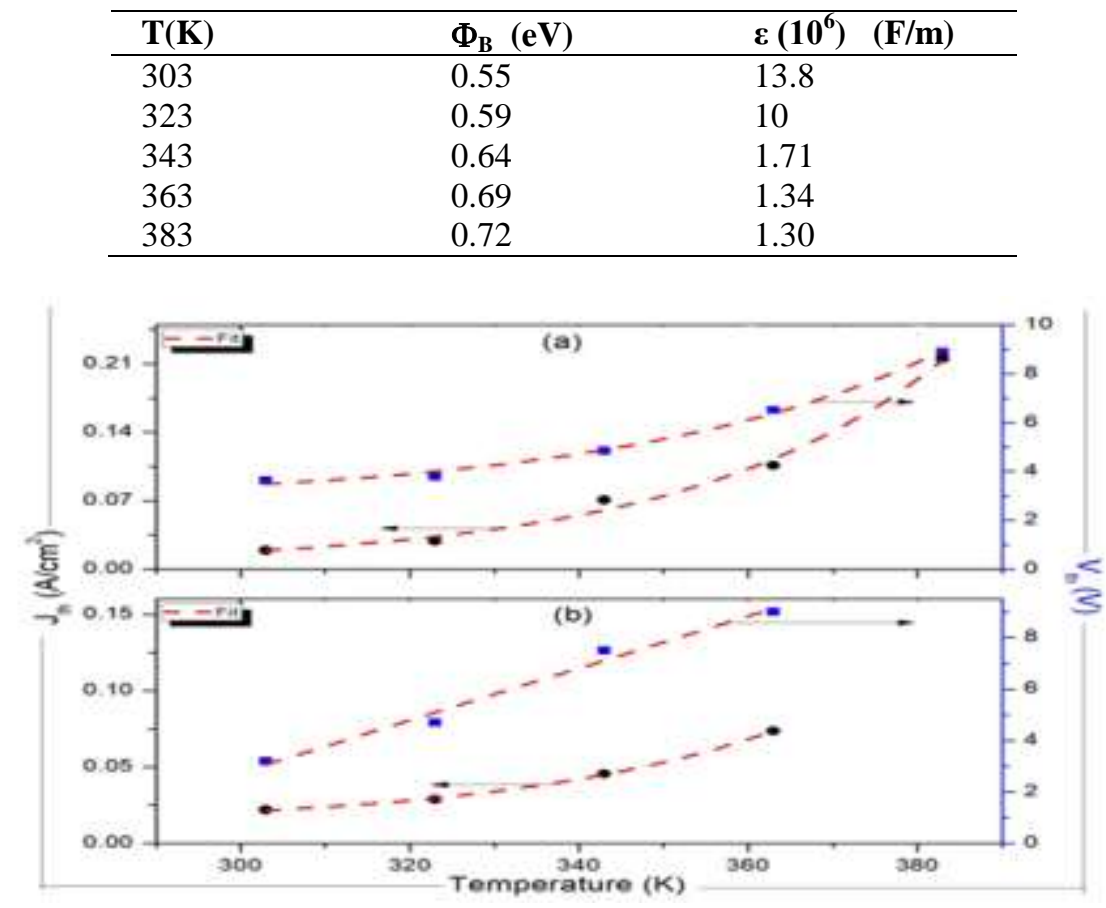

Fig (9) Temperature dependence of threshold voltage and current density at turnover point at (a) forward, (b) reverse directions for $\mathrm{Cu} / \mathrm{PEDOT}: \mathrm{PSS} / \mathrm{Cu}$ device. 
Mansy

The dependence of hysteresis area $\left(\mathrm{A}_{\text {hyst }}\right)$ involved in both forward direction $\left(\mathrm{A}_{\text {hyst-F}}\right)$ and reverse direction $\left(\mathrm{A}_{\text {hyst- }}\right.$ $R$ ) in both polarities between 0 to $\pm 15 \mathrm{~V}$ are obtained at different temperatures, Fig (10a). The hysteresis area in both two polarities means energy stored in the polymer matrix. This difference in hysteresis area in both forward and reverse directions with temperature depends on structure, disorder level and the change in morphology of PEDOT:PSS with temperature.

The relative variation of hysteresis area $\left(\left(\mathrm{A}_{\text {hyst } \mathrm{R}} / \mathrm{A}_{\text {hyst- }}\right.\right.$ $\left.\left.{ }_{F}\right)=A_{R} / A_{F}\right)$ has been estimated and plotted against ambient temperature $(\mathrm{T})$. It is noticed that $\mathrm{A}<1$, decreases linearly with increasing temperatures $(A=-0.009 \mathrm{~T}+3.5)$, Fig (10b).
The area in the forward direction (attributed to polarization) is larger than that of the reverse direction (attributed to depolarization). This means that the reverse direction needs some energy to reset the polarization gained in the forward direction and some energy to get polarization in the opposite polarities. However, the amount of energy stored in the reverse direction appeared to be smaller than that in the forward direction.

Fig (11), illustrates the effect of scan rate on J-V characteristics curves at room temperature for $\mathrm{Cu} / \mathrm{PEDOT}: \mathrm{PSS} / \mathrm{Cu}$ device as a representative diagram. All scan rates show reversible a unipolar resistive switching memory.

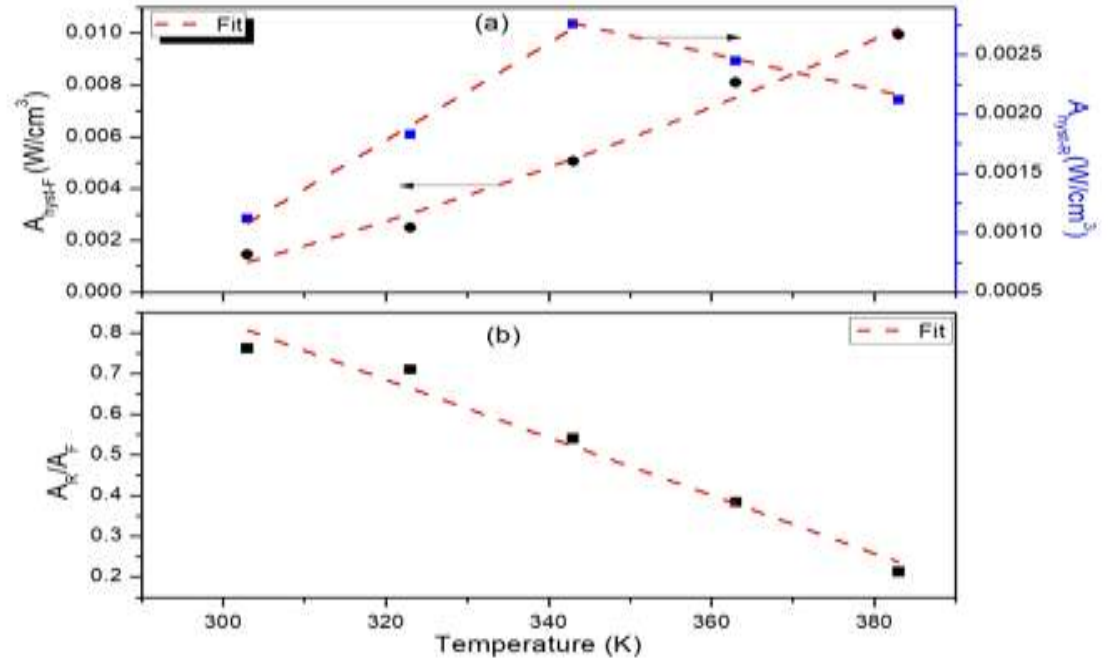

Fig (10) Temperature dependence of hysteresis area (a) forward and reverse directions (b) the ratio of reverse to forward area for $\mathrm{Cu} / \mathrm{PEDOT}: \mathrm{PSS} / \mathrm{Cu}$ device.

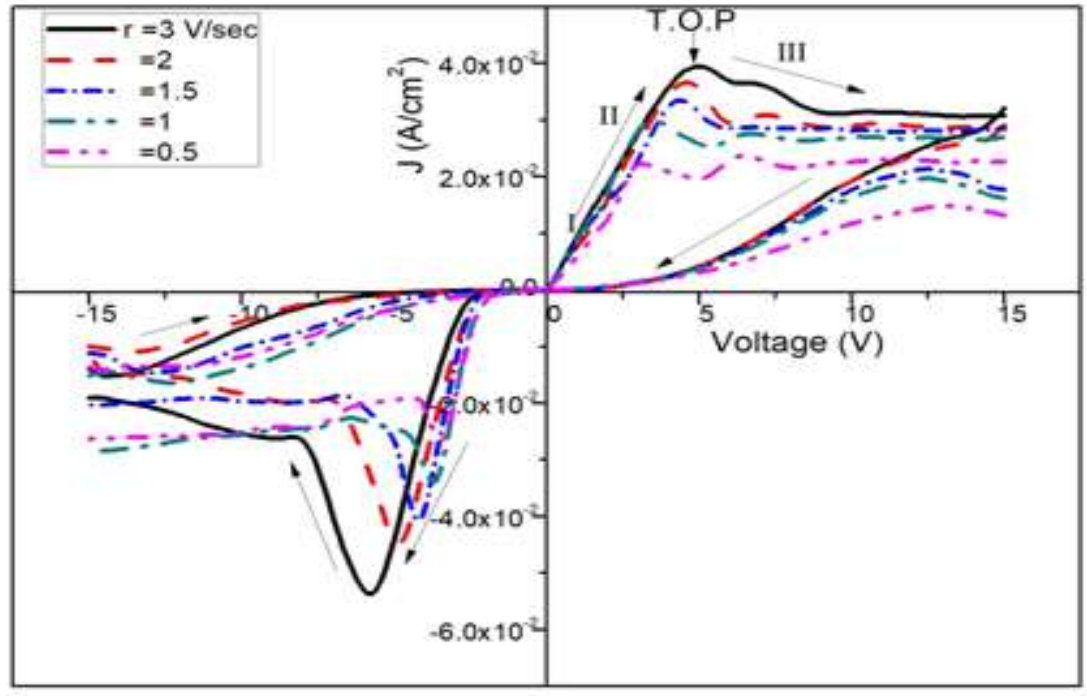

Fig (11) J-V characteristic curves of a device at different scan rate for Cu/PEDOT:PSS/Cu device. 
It is noticed that, the turnover point is shifted towards higher threshold voltage and higher threshold current density as the scan rate is increased, Fig (12).

The increasing of $V_{\text {th }}$ and $J_{\text {th }}$ with increasing the scan rate may be related to the charge injection/extraction by the electrodes[27,28]. In addition, the polarization/ depolariza- tion are dependence on the scan rates. The hysteresis area increases with increasing the scan rate, Fig (13a). In addition, the hysteresis area in the reverse direction $\mathrm{A}_{\text {hyst- } \mathrm{R}}$ is larger than hysteresis area in forward direction $\mathrm{A}_{\text {hyst-F, Fig }}$ (13b).

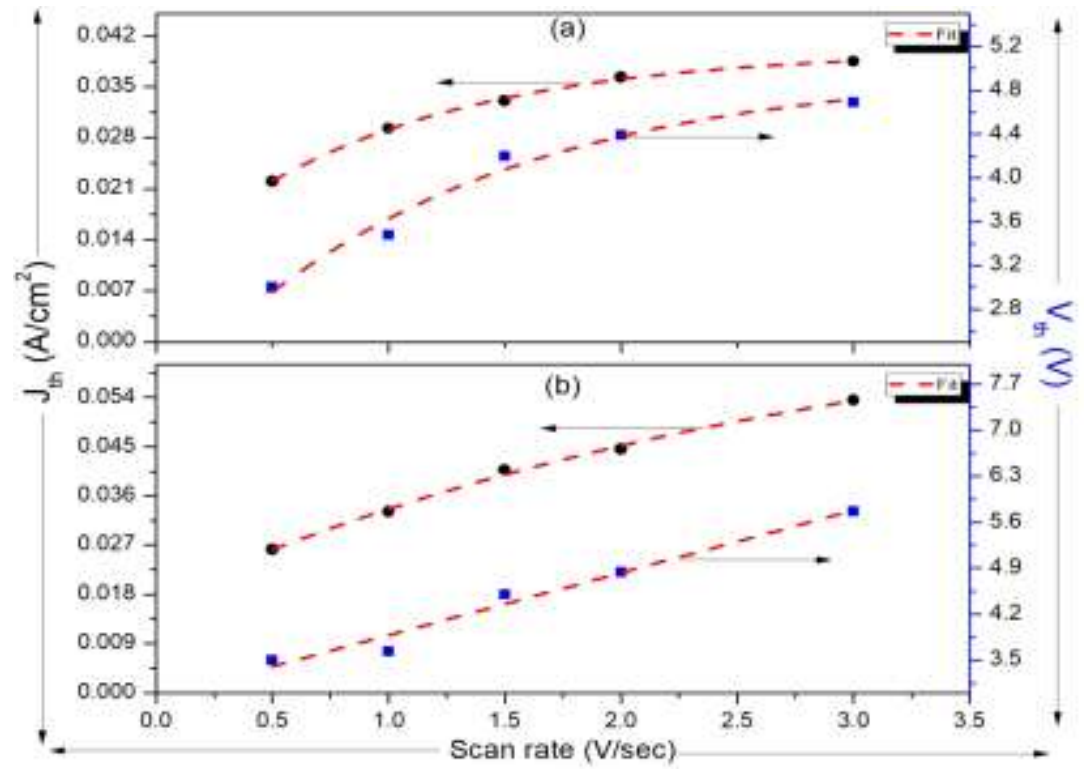

Fig (12) Scan rate dependence of threshold voltage and current density at turnover point at (a) forward, (b) reverse directions for $\mathrm{Cu} / \mathrm{PEDOT}: \mathrm{PSS} / \mathrm{Cu}$ device.
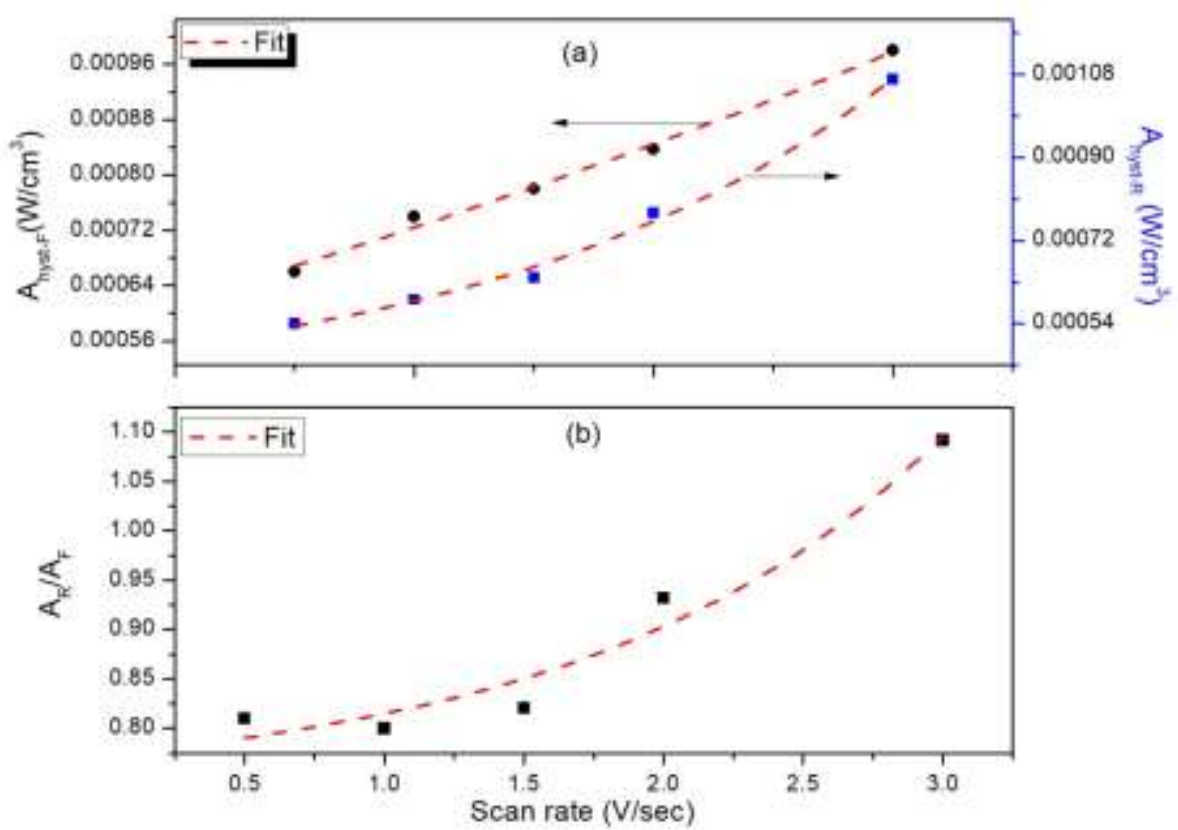

Fig (13) Scan rate dependence of hysteresis area, (a) forward and reverse directions (b) the ratio of reverse to forward directions area for $\mathrm{Cu} / \mathrm{PEDOT}$ :PSS/Cu device. 
Mansy

\section{Conclusion}

From the present study, the following conclusion has been extracted,

1-The study of frequency dependence ac conductivity under different temperatures indicated ionic and electronic components in the conduction process. The dominant conduction mechanism in PEDOT:PSS film is (OLPT) model

2-The study of J-V characteristic of PEDOT:PSS film exhibits an anomalous behavior with turnover at a characteristic point. This point depends on both the ambient temperature and delay time. It is found that both the threshold current density, the voltage at the turnover point, and the hysteresis area increase with increasing both (temperatures and scan rate). The analysis of results enables us to explain the carrier transport in region II by thermionic emission whereas in region III (NDR) the behavior is attributed to polarization. In the reverse direction, the behavior is attributed to depolarization.

\section{References}

[1] N. Saxena, M. Čorić, A. Greppmair, J. Wernecke, M. Pflüger, M. Krumrey, M.S. Brandt, E.M. Herzig, P. Müller-Buschbaum, Morphology-Function Relationship of Thermoelectric Nanocomposite Films from PEDOT:PSS with Silicon Nanoparticles, Adv. Electron. Mater. Vol.3, PP. 1-14, 2017. https://doi.org/10.1002/aelm.201700181.

[2] B. Fan, X. Mei, J. Ouyang, Significant conductivity enhancement of conductive poly $(3,4-$ ethylenedioxythiophene): Poly(styrenesulfonate) films by adding anionic surfactants into polymer solution, Macromolecules. Vol.41, PP. 5971-5973, 2008. https://doi.org/10.1021/ma8012459.

[3] D. R. Lamb, Electrical Conduction Mechanisms inThin Insulating Films, Methuen, London, UK, 1967.

[4] F. Chiu, A Review on Conduction Mechanisms in Dielectric Films, Adv. Mater. Sci. Eng. Vol.2014, PP.1-14, 2014.

[5] E.W. Lim, R. Ismail, Conduction mechanism of valence change resistive switching memory: A survey, Electron. Vol.4, PP. 586-613, 2015. https://doi.org/10.3390/electronics4030586.

[6] V.C. Nguyen, P.S. Lee, Resistive Switching Memory Phenomena in PEDOT PSS: Coexistence of Switchable Diode Effect and Write Once Read Many Memory, Sci. Rep. Vol.6, PP. 1-7, 2016. https://doi.org/10.1038/srep19594.

[7] H.Y. Jeong, J.Y. Kim, T.H. Yoon, S.Y. Choi, Bipolar resistive switching characteristics of poly(3,4ethylene-dioxythiophene): Poly(styrenesulfonate) thin film, Curr. Appl. Phys. Vol.10, PP. e46-e49, 2010. https://doi.org/10.1016/j.cap.2009.12.011.

[8] Z.S. Wang, F. Zeng, J. Yang, C. Chen, Y.C. Yang, F. Pan, Reproducible and controllable organic resistive memory based on $\mathrm{Al} /$ poly(3,4-ethylenedioxythiophene):poly(styrenesulfonate)/Al structure,
Appl. Phys. Lett. Vol.97, PP. 2008-2011, 2010. https://doi.org/10.1063/1.3529455.

[9] J.Y.J.W. Kim, H.Y. Jeong, J.Y.J.W. Kim, T.H. Yoon, S.Y. Choi, Critical role of top interface layer on the bipolar resistive switching of Al/PEDOT:PSS/Al memory device, Curr. Appl. Phys. Vol.11, PP. e35e39 , 2011. https://doi.org/10.1016/j.cap.2010.12.038.

[10] H. Ha, O. Kim, Unipolar switching characteristics of nonvolatile memory devices based on $\operatorname{poly}(3,4-$ ethylenedioxythiophene):Poly(styrene sulfonate) thin films, Jpn. J. Appl. Phys. Vol.48, PP. 4-7, 2009. https://doi.org/10.1143/JJAP.48.04C169.

[11] H. Ha, O. Kim, Electrode-Material-Dependent Switching Characteristics of Organic Nonvolatile Memory Devices Based on, Film. Vol.31, PP. 368$370,2010$.

[12] H. Ha, O. Kim, Bipolar switching characteristics of nonvolatile memory devices based on $\operatorname{poly}(3,4-$ ethylenedioxythiophene):poly(styrenesulfonate) thin film, Appl. Phys. Lett. Vol.93, PP. 1-3, 2008. https://doi.org/10.1063/1.2960998.

[13] X.N. Xie, K.K. Lee, X. Chen, K.P. Loh, C.H. Sow, A.T.S. Wee, New scenarios of charge transport in PEDT:PSS conducting polymer: From hole resonant tunneling to cationic motion and relaxation, Org. Electron. Vol.11, PP. 1432-1438, 2010. https://doi.org/10.1016/j.orgel.2010.05.013.

[14] I. Salaoru, C.C. Pantelidis, Electrical re-writable nonvolatile memory device based on PEDOT:PSS thin film, Micromachines. Vol.11, PP. 1-7, 2020. https://doi.org/10.3390/mi11020182.

[15] Q. Zhu, E. Yildirim, X. Wang, X.Y.D. Soo, Y. Zheng, T.L. Tan, G. Wu, S.W. Yang, J. Xu, Improved Alignment of PEDOT:PSS Induced by in-situ Crystallization of "Green" Dimethylsulfone Molecules to Enhance the Polymer Thermoelectric Performance, Front. Chem. Vol.7, PP. 1-11, 2019. https://doi.org/10.3389/fchem.2019.00783.

[16] E. Hosseini, V. Ozhukil Kollath, K. Karan, The key mechanism of conductivity in PEDOT:PSS thin films exposed by anomalous conduction behaviour upon solvent-doping and sulfuric acid post-treatment, J. Mater. Chem. C. Vol.8, PP.3982-3990, 2020. https://doi.org/10.1039/c9tc06311k.

[17] X. Wang, K. Ko, C. Yin, F. Wang, Q. Zhu, T. Tang, Enhancement of thermoelectric performance of PEDOT: PSS fi $1 \mathrm{~ms}$ by post-treatment with a superacid, RSC Adv. Vol.8, PP. 18334-18340, 2018. https://doi.org/10.1039/C8RA02058B.

[18] K.E. Aasmundtveit, E.J. Samuelsen, L.A.A. Pettersson, O. Inganäs, T. Johansson, R. Feidenhans'l, Structure of thin films of $\operatorname{poly}(3,4-$ ethylenedioxythiophene), Synth. Met. Vol.101, PP.561-564, 1999. https://doi.org/10.1016/S0379-6779(98)00315-4.

[19] J. Zhou, D.H. Anjum, L. Chen, X. Xu, I.A. Ventura, L. 
Jiang, G. Lubineau, The temperature-dependent microstructure of PEDOT/PSS films: insights from morphological, mechanical and electrical analyses, J. Mater. Chem. C. Vol.2, PP. 9903-9910, 2014. https://doi.org/10.1039/C4TC01593B.

[20] A.K. Jonscher, Dielectric relaxation in solids, J. Phys. D. Appl. Phys. Vol.32, 1999. https://doi.org/10.1088/0022-3727/32/14/201.

[21] K.S. Gilkoy, W.A. Phillips, An asymmetric doublewell potential model for structural relaxation processes in amorphous materials, Philos. Mag. B Phys. Condens. Matter; Stat. Mech. Electron. Opt. Magn. Prop. Vol.43, PP. 735-746, 1981. https://doi.org/10.1080/01418638108222343.

[22] A.R. Long, Frequency-dependent loss in amorphous semiconductors, Adv. Phys. Vol.31, PP. 553-637, 1982.

[23] A. Ghosh, Transport properties of vanadium germanate glassy semiconductors, Phys. Rev. B. Vol.42, PP.5665-5676, 1990. https://doi.org/10.1103/PhysRevB.42.5665.

[24] M.F. Kotkata, F. Abdel-Wahab, F.A. Abdel-Wahab, H.M. Maksoud, Investigations of the conduction mechanism and relaxation properties of semiconductor Sm doped a-Se films, Artic. J. Phys. D Appl. Phys. Vol.39, PP.2059-2066, 2006. https://doi.org/10.1088/0022-3727/39/10/013.

[25] Nora.E, Dielectric properties and molecular behaviour, London, New York, Van Nostrand Reinhold, 1969.

[26] S.D. Druger, A. Nitzan, M.A. Ratner, Dynamic bond percolation theory: A microscopic model for diffusion in dynamically disordered systems. I. Definition and one-dimensional case, J. Chem. Phys. Vol.79, PP.3133-3142, 1983. https://doi.org/10.1063/1.446144.

[27] X.N. Xie, X. Gao, Y. Wang, J. Wang, K.P. Loh, A.T.S. Wee, Negative differential resistance based on electron injection/extraction in conducting organic films, Appl. Phys. Lett. Vol.95, PP.10-13, 2009. https://doi.org/10.1063/1.3202414.

[28] R.N. Bhowmik, K.V. Siva, Non-equilibrium character of resistive switching and negative differential resistance in Ga-doped Cr2O3 system, J. Magn. Magn. Mater. Vol.457, PP. 17-29, 2018. https://doi.org/10.1016/j.jmmm.2018.02.070. 